\title{
Low-Voltage-Driven Fabrication of a High-Luminance Multi-Layered Green OLED
}

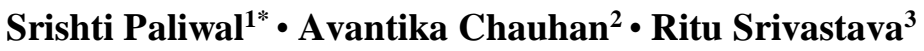 \\ ${ }^{1}$ School of Materials Science and Technology, Indian Institute of Technology (BHU), Varanasi (221005), Uttar \\ Pradesh, India. \\ ${ }^{2}$ Department of Physics, Dev Bhoomi Group of Institutions, Dehradun (248007), Uttarakhand, India. \\ ${ }^{3}$ Advanced Materials and Devices Metrology Division, CSIR-National Physical Laboratory, New Delhi \\ (110012) India.
}

*Corresponding author Email: srishtiuk77@gmail.com

Received: 29.8.2021; Revised: 18.11.2021; Accepted:26.11.2021

(C)Society for Himalayan Action Research and Development

\begin{abstract}
This work presents a five-layered Organic Light Emitting Device (OLED) fabricated using the stacked configuration of LiF/Alq3/CBP-Ir (ppy) 3/ $\alpha$-NPD/F4-TCNQ by the Vacuum Thermal Evaporation Technique. A Keithley 2400 source meter was used for I-V characterization, and a Luminance meter (LMT L1009) was used for L-V characterization. The I-V characterization findings demonstrate that at low voltages of about 10 volts, the OLED emits neonish green light, which is correlated to the type of organic material used as an emissive layer, however as the voltage is increased further, the current increases, exhibiting nonlinearity up to 20 volts. Further increments in voltage do not achieve the desired outcomes, but instead produce heat as a result of non-radiative processes such as thermal relaxation of excitons, photon absorption, or internal reflection, which are undesirable and shorten the life of a device. Pixels were completely burned off after a certain amount of applied voltage was reached. The device yielded the highest luminance of 30551cd.m-2 at 20 volts, according to the $\mathrm{L}-\mathrm{V}$ characterization results.
\end{abstract}

Keywords: Organic Light Emitting Device, I-V, L-V, Luminance

\section{Introduction}

\section{Organic light-emitting diodes (OLEDs) are} contemplated as one of the most remarkable and noticeable next-generation display technologies that emerged over the last few decades, alluring a lot of attention from all over the world owing to their promising applications in the multi-diverse discipline. These are the elementary solid-state semiconductor devices working on the basic principle of electroluminescence $(\boldsymbol{E L})$.

(Braun and Heeger 1991, Greenham and Friend 1995, Pope et al. 1963) An expansive range of electroluminescent organic semiconductors for charge transporters, emitters, and dopants provide a plethora of options to choose from, breeding numerable possible structures with color selectivity prerogative. (ĩmija et al. 2006) An OLED with a single-layer configuration has poor efficiency, poor brightness, and a high driving voltage. The enhancement of external quantum efficiency close to one percent and the reduction in the driving voltage close to 10 volts can be obtained by employing a binary layer structure composed of distinct organic 
materials. (Tang 1982, Tang and VanSlyke 1987) Within the bulk, electron and hole recombination takes place due to the presence of the charge blocking layer which helps in minimizing non-radiative losses. Charge blocking layers, according to previous research, increase the performance of organic phosphorescent light-emitting diodes by $19 \%$ on average by transforming triplet excitons into usable radiation, and fluorescent lightemitting diodes by $8 \%$. They are superior for next-generation display technology due to properties such as self-luminescence, flexibility, low power consumption, ease of fabrication, wide viewing angle, lightweight, and compaction.

\section{Material and Methods}

\section{Device Structure and Material Used for Synthesis}

Figure 1 depicts the simple ordered stacked unit layout of a five-layered OLED that was used.

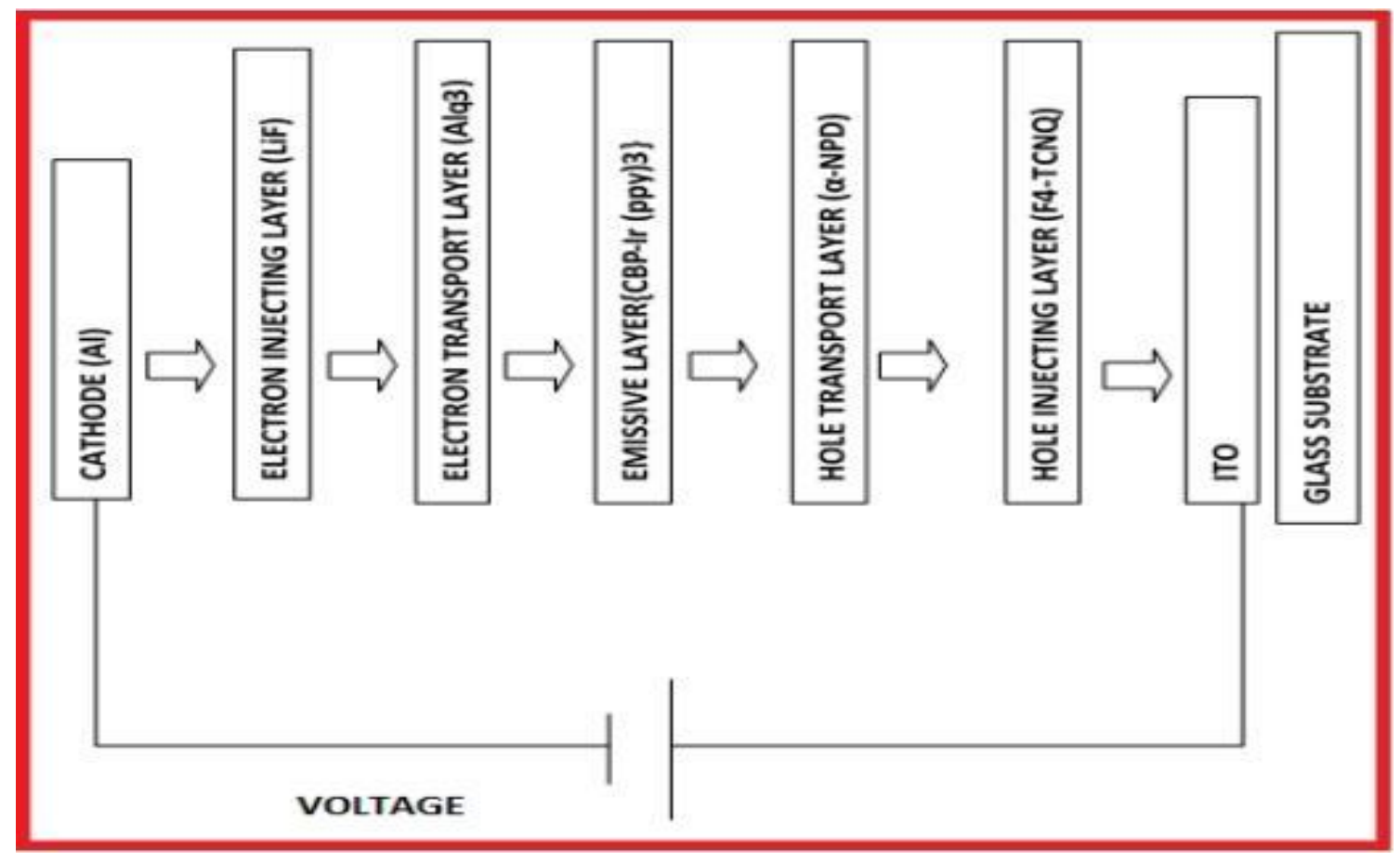

Fig. 1. A five-layered Organic Light Emitting Diode's heterogeneous network structure.

The device primarily incorporates five layers along with cathode, anode, and glass substrate each essentially accomplishing their specific function. Materials play a pivotal role in both the efficiency and the longevity of the device. [Hung and Chen 2002] Because of its high work function and good transparency in the visible spectrum, an indium tin oxide (ITO) coated glass substrate was used as an anode component. At the HOMO level of organic materials, the insertion of holes can be encouraged by using ITO. Aluminum (Al) was used as a cathode to preserve the disparity between the work functions of electrodes, facilitating the injection of electrons into the LUMO level of an organic layer. Alq3 \{Tris- 
(8-hydroxyquinoline) aluminum $\}$ was used as an electron transport layer (ETL) because of its high electron affinity and mobility, keeping

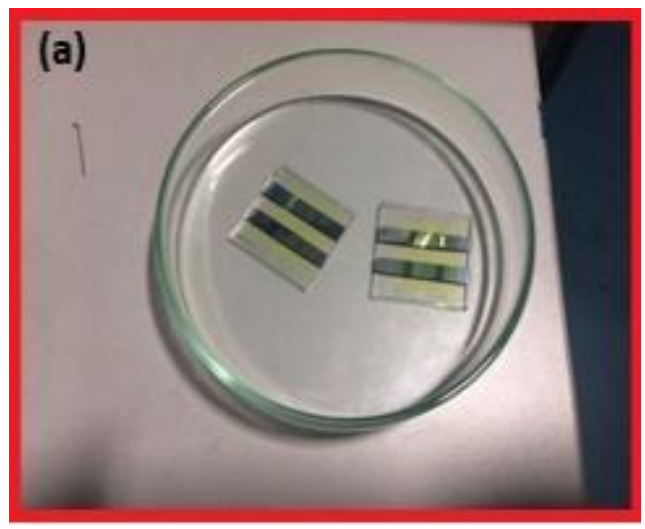

Fig. 2. (a) Fabricated OLED device

Due to possession of high hole mobility and high glass transition temperature N, N'-Di-((1naphthyl)-N, N'-diphenyl)-1, 1'-biphenyl)-4, 4'-diamine namely $\alpha$-NPD was used as a hole transport layer (HTL) while $\mathrm{F}_{4}$-TCNQ $(2,3,5$, 6,-tetrafluoro-7, $\quad 7, \quad 8, \quad 8$ tetracyanoquinodimethane) was used as a hole-injecting layer.[Kalinowski 1999, Gao and Kahn 2003] CBP :Ir (ppy) 3and LiF were used as an emissive layer and an electron injecting material, respectively. [Sanderson et al. 2019]

\section{Device Fabrication Method}

The physical technique chosen for the deposition of the thin film was Vacuum Thermal Evaporation due to its simplicity, economic benefit, and less damaging behavior towards the substrate. To prevent the vaporatmosphere reaction lower pressures of the order of $10^{-6}$ were used. [Glang 1970] Prior to deposition, the ITO-coated glass substrate was holes "blocked" from passing. [Kulkarni et al. 2004]

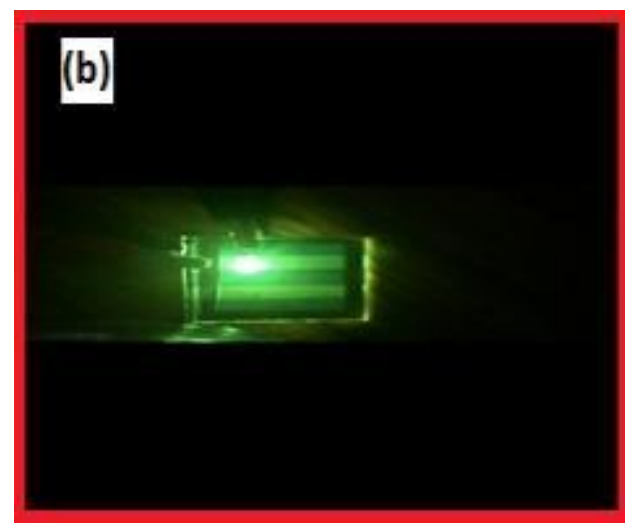

(b) OLED while emitting green light patterned with a laser scribing method, followed by a rigorous cleaning process that included first a soap solution, then ultrasonication in DI (deionized) water for 45 minutes, with the DI water being alternated every 15 minutes. Cleaning the substrate in acetone and isopropanol baths took 15 minutes each. The substrate in the Teflon stand was then immersed in isopropanol and put on a hot plate to boil, after which it was sprayed with steam. Afterward, the cleaned substrate was baked in a vacuum oven to remove any remaining impurities. Table 1 illustrates the experimental specifications of vacuum thermal deposition comprising various parameters not to mention processing variables such as deposition rate, geometry, degree of vapor and gaseous contamination in the atmosphere, and substrate temperature should be taken keen care of. Figure 2 (a) shows the assembled device, while figure 2 (b) shows the device illuminated with a neonish green light. 
Table 1: Summary of experimental details showing vacuum level, deposition rate, thickness monitor reading, and corresponding values of current

\begin{tabular}{|c|c|c|c|c|}
\hline Material used & $\begin{array}{l}\text { Vacuum Level } \\
\text { (mbar) }\end{array}$ & $\begin{array}{l}\text { Deposition rate } \\
(\AA / \mathrm{A} / s)\end{array}$ & $\begin{array}{l}\text { Thickness } \\
\text { (nm) }\end{array}$ & Current (A) \\
\hline F4-TCNQ & $3 \times 10^{-6}$ & $0.1-0.2$ & 4 & $0.65-0.73$ \\
\hline$\alpha-N P D$ & $3 \times 10^{-6}$ & $0.2-1.4$ & 30 & $1.75-2.22$ \\
\hline CBP:Ir(ppy)3 & $3 \times 10^{-6}$ & $0.2-0.7$ & 35 & $0.25-0.83$ \\
\hline Alq3 & $3 \times 10^{-6}$ & $0.2-0.8$ & 28 & $0.45-1.50$ \\
\hline $\mathrm{LiF}$ & $3 \times 10^{-6}$ & 0.1 & 1 & 2.3 \\
\hline $\mathrm{Al}$ & $2 \times 10^{-6}$ & $2-4$ & 170 & $0.53-0.64$ \\
\hline
\end{tabular}

\section{Results}

Current-Voltage Characteristics

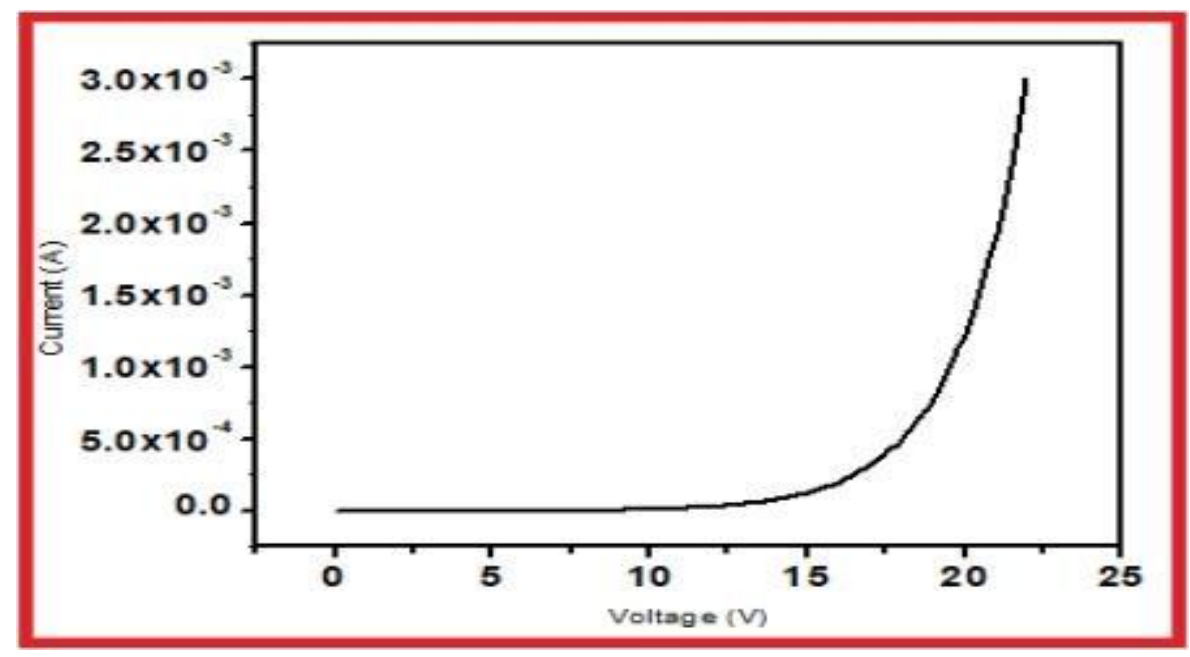

Fig. 3. A typical I-V characteristic for a prepared five-layered organic light-emitting diode.

The I-V characterization was performed with a

Keithley 2400 source meter, which indicates that the current remains zero until the voltage exceeds the potential barrier that exists between the electron transport layer (ETL) and the hole transport layer (HTL). The OLED system did not emit any light up to 5 volts due to space charge effects, but as we steadily increased the voltage above 10 volts, the onset of electroluminescence began, and above this voltage, the current escalated non-linearly, resulting in the emission of the bright neonish green light shown in figure 2 (b). If the voltage is increased up to 20 volts, the intensity of the light produced by the OLED increases. Increasing the voltage further does not yield any useful results; instead, it induces heat due to non-radiative processes such as thermal relaxation of excitons, photon absorption, or internal reflection, which are undesirable and decrease the device's lifespan. The pixels were totally burned out at 28 volts. The output light intensity or brightness is directly proportional to the amount of applied electrical current. 


\section{Luminance-Voltage Characteristics}

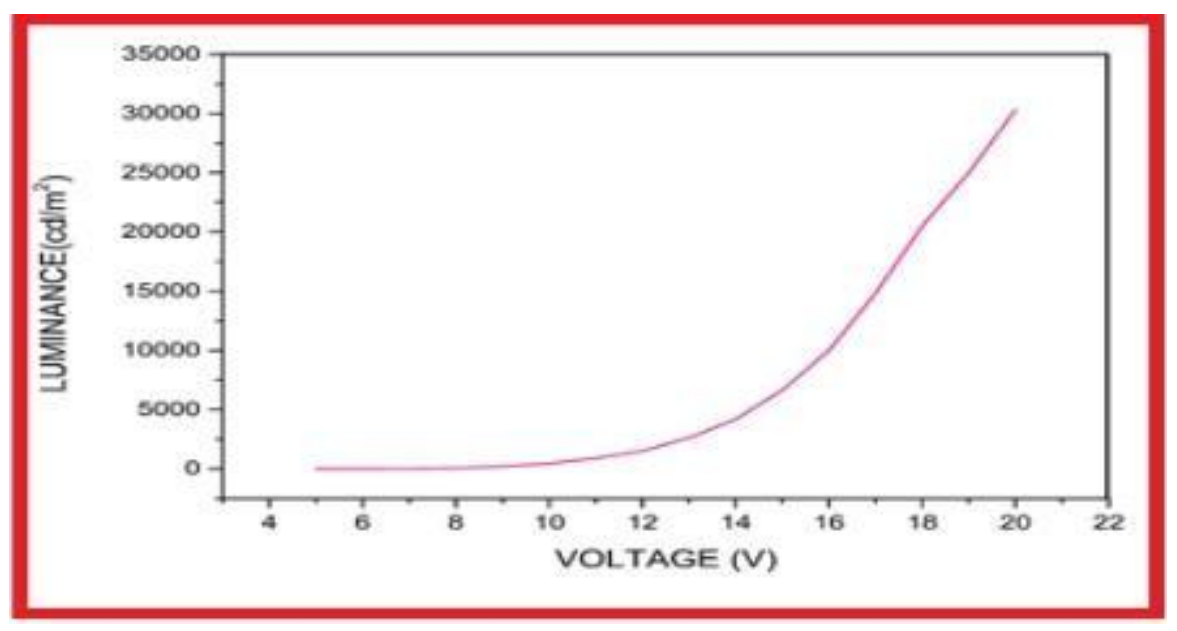

Fig. 4. A typical luminance versus voltage plot for the prepared organic light-emitting diode.

A Luminance meter (LMT L1009) is used to perform an $\mathrm{L}-\mathrm{V}$ characterization, which is a photometric approximation of the luminosity of light moving in a specific direction per unit area. It is worth noting that the beginning of luminance can be seen when the voltage exceeds the magnitude of 10 and that this contributes to non-linear luminance activity as the voltage increases. At $20 \mathrm{~V}$, the device generated the highest luminance of 30300 cd.m-2. An EL spectrum can be obtained using high-resolution spectrometer characterization. [Kumar et al. 2010] Using the energy wavelength relationship, the bandgap can be calculated using the wavelength corresponding to the highest peak observed in the EL spectrum. The wavelength of the light emitted is a characteristic of the organic molecules involved in the formation of an emissive layer.

\section{Discussion}

For continuous exciton formation in OLEDs, synchronized insertion of electrons and holes in the electroluminescent emissive organic layer is needed. [Tang, Vanslyke and Chen 1989] A five-layered OLED was designed to ensure proper functioning and efficiency. The device began emitting neonish green light onset starting as early as around 10 volts, ensuring low driving voltage, and reached a max luminance yield of 30300 cd.m-2at 20 volts. Thus, the additional layers aid in providing a simultaneous supply of both charge carriers, reducing non-radiative losses caused by charge dumped via the thermal relaxation of excitons, photon absorption, or internal reflection, which will result in better overall efficiency of the device.

\section{Acknowledgements}

I wish to extend my sincere gratitude to the Council of scientific and industrial- National Physical Laboratory, New Delhi for enabling me to use the lab facilities for my research, as well as to everyone who has assisted me along the way. 


\section{References}

Braun D and Heeger A (1991),'Visible light emission from semiconducting polymer diodes", Applied Physics Letters 58, 1982. https://doi.org/10.1063/1.105039

Gao W and Kahn A (2003), "Controlled p doping of the hole-transport molecular material N, N'- diphenyl-N,N'-bis(1naphthyl)-1,1'-biphenyl-4,4'-diamine, $\mathrm{N}^{\prime}$ diphenyl-N,N'-bis(1-naphthyl)- $\quad$ 1,1'biphenyl-4,4'-diamine with tetrafluorotetracyanoquinodimethane", Journal of Applied Physics 94, 359. https://doi.org/10.1063/1.1577400

Glang M. (1970) Handbook of thin film technology. McGraw-Hill.

Greenham NC and Friend RH (1995) "In Solid State Physics", Academic Press, New York, London, 2- 150.

Hung LS and Chen CH (2002)," Recent progress of molecular organic electroluminescent materials and devices", Materials Science and Engineering R 39 143-222.

ĩmija J, Maáachowski MJ, ZieliĚski J, Wacáawek M, ĝciē̄ka K (2006)“'Organic materials for electronics", Chemistry Didactics, Ecology-Metrology 11/1-2 69-80 (in Polish)

Kalinowski J (1999),"Electroluminescence in organics", Journal of Physics D: Applied Physics 32 179-250.

Kulkarni AP,Christopher J. Tonzola, Babel A, and Samson A. Jenekhe(2004), "Electron Transport Material for Organic Light-
Emitting Diode", Chemistry of Materials 16 (23), 4556-4573; https://doi.org/10.1021/cm0494731

Kumar R, Srivastava R, Kumar A, Kamalasanan MN and Singh K (2010). Green-light-emitting electroluminescent device based on a new cadmium complex. EPL (Europhysics Letters), 90(5), 57004. http://dx.doi.org/10.1209/0295$\underline{5075 / 90 / 57004}$

Pope M, Kallmann HP, and Magnate P (1963) "Electroluminescence in Organic Crystals", The Journal of Chemical Physics 38, 2042-2043 https://doi.org/10.1063/1.1733929

Sanderson S, Philippa B, Vamvounis G, Burn PL, White RD (2019), “Understanding charge transport in Ir (ppy)3: CBP OLED films",Journal of Chemical Physics. 150(9):094110. https://doi.org/10.1063/1.5083639

Tang CW (1982) "Organic electroluminescent cell", US Patent 4356429

Tang CW, Vanslyke SA and Chen CH (1989). "Electroluminescence of doped organic thin films", J Applied Physics 65,3610; https://doi.org/10.1063/1.343409

Tang CW, VanSlyke SA (1987), "Organic electroluminescent diodes", Applied Physics Letters 51, 913; $\underline{\text { https://doi.org/10.1063/1.98799 }}$ 